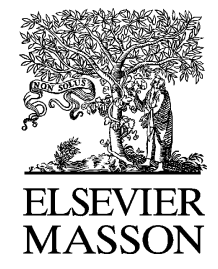

\title{
Conférence
}

\section{Imagerie phénotypique et peptides radiomarqués au gallium-68 : au-delà des analogues de la somatostatine}

\author{
Phenotypic imaging and gallium-68 radiolabeled peptides: Beyond the somatostatin analogs \\ O. Couturier ${ }^{\mathrm{a}, *, \mathrm{~b}}$, F. Lacoeuille ${ }^{\mathrm{a}, \mathrm{b}}$, C. Lefebvre ${ }^{\mathrm{a}, \mathrm{b}}$, F. Hindre ${ }^{\mathrm{a}, \mathrm{b}}$, L. Vervueren $^{\mathrm{a}, \mathrm{b}}$, \\ F. Bouchet ${ }^{\mathrm{a}, \mathrm{b}}$, J.-J. Le Jeune ${ }^{\mathrm{a}, \mathrm{b}}$, R. Hustinx ${ }^{\mathrm{c}}$ \\ ${ }^{a}$ Service de médecine nucléaire universitaire, université d'Angers, 4, rue Larrey, 49933 Angers cedex 9, France \\ ${ }^{\mathrm{b}}$ Inserm U646, université d'Angers, 49100Angers, France \\ c Département de médecine nucléaire universitaire, domaine universitaire du Sart Tilman, Liège, Belgique \\ Reçu le 22 février 2010 ; accepté le 23 février 2010 \\ Disponible sur Internet le 2 avril 2010
}

\section{Résumé}

Le ciblage de récepteurs avec des peptides radiomarqués est devenu très important en oncologie nucléaire au cours de ces dernières années. Les peptides les plus fréquemment utilisés en clinique sont les analogues de la somatostatine. Cependant, d'autres analogues radiomarqués ont également été développés et évalués in vitro et in vivo et pour certains déjà en clinique. Il s'agit par exemple de l'alpha-melanocyte-stimulating hormone (alpha-MSH), de la neurotensine, du peptide intestinal vasoactif (VIP), de la bombésine, de la substance P (SP) et de la cholécystokinine (CCK), des intégrines... Cette revue se focalise sur les peptides radiomarqués au gallium-68, développés pour l'imagerie TEP, en dehors des analogues de la somatostatine, qui sont discutés dans un autre article.

(C) 2010 Elsevier Masson SAS. Tous droits réservés.

Mots clés : Gallium-68 ; Peptides ; Cancer ; Tomographie par émission de positons ; TEP ; Imagerie moléculaire ; Imagerie phénotypique

\section{Abstract}

Receptor targeting with radiolabeled peptides has become very important in nuclear oncology in the past few years. The most frequently used peptides in the clinic are analogs of somatostatin. However, other radiolabeled analogs have also been developed and assessed in vitro and in vivo and some of them are already in clinical use. For instance, radiolabeled analogs of alpha-melanocyte-stimulating hormone (alpha-MSH), neurotensin, vasoactive intestinal peptide (VIP), bombesin (BN), substance P (SP), cholecystokinin (CCK), integrins. . . This review focuses on gallium-68 radiolabeled peptides developed for PET imaging, except for somatostatin analogs, which are discussed in another article.

(C) 2010 Elsevier Masson SAS. All rights reserved.

Keywords: Gallium-68; Peptides; Cancer; Positron emission tomography; PET; Molecular imaging; Phenotypic imaging

\section{Introduction}

Le développement récent de la tomographie par émission de positons avec du gallium-68 ( ${ }^{68} \mathrm{Ga}$-PET) est lié à la commercialisation de générateurs gallium-68/germanium- $68\left({ }^{68} \mathrm{Ga} /{ }^{68} \mathrm{Ge}\right)$, utilisables en recherche préclinique même sans autorisation de mise sur le marché (AMM) et à la chimie du gallium permettant

\footnotetext{
* Auteur correspondant.

Adresse e-mail : ocouturier70@me.com (O. Couturier).
}

de chélater diverses molécules et des analogues spécifiques de récepteurs cellulaires. Des générateurs ${ }^{68} \mathrm{Ga} /{ }^{68} \mathrm{Ge}$ « GMP » (good managment practise) devraient être disponibles dans un avenir proche en France, étape décisive du processus d'AMM avant une distribution par les industriels de la radiopharmacie aux services de médecine nucléaire pour la pratique clinique.

Le gallium-68 est particulièrement attractif pour les départements de médecine nucléaire qui n'ont pas d'accès direct au fluor-18 des cyclotrons. Il n'est, en effet, pas envisageable d'attendre une à deux heures de décroissance pendant le transport de fluor-18 avant l'étape de radiosynthèse, 
alors que les rendements de radiosynthèse de nombreux traceurs TEP, autres que le FDG, sont déjà souvent peu élevés. Ainsi, le générateur ${ }^{68} \mathrm{Ge} /{ }^{68} \mathrm{Ga}$ permettrait de disposer sur place et en permanence d'un émetteur de positons (gallium-68 : demi-vie 68 minutes, $89 \%, \mathrm{E}_{\beta+}=1,90 \mathrm{MeV}$ ) dans les services de médecine nucléaire (durée moyenne d'utilisation de six mois environ pour les générateurs qui pourraient être autorisés en France, grâce à la longue demi-vie de 270,8 jours du parent germanium-68).

La dernière décennie a vu le développement rapide de peptides radiomarqués, pour des applications diagnostiques et/ ou thérapeutiques, en raison de méthodes simplifiées de purification, de la facilité de production à moindre frais de ces molécules de petite taille, de leur pénétration rapide dans les tissus et de leur faible antigénicité. Le gallium-68 est particulièrement adapté au radiomarquage de nombreux peptides ; le chélateur de choix est l'acide 1,4,7,10-tétraazacyclodecane-1,4,7,10-N,N',N",N"',-tétra-acetique (DOTA) avec lequel il est possible de former des complexes stables de gallium-68, utilisés avec succès en préclinique et parfois même en clinique pour l'imagerie TEP. La plupart des efforts de développement clinique ont porté sur l'imagerie TEP des récepteurs de la somatostatine avec des analogues DOTAgallium de la somatostatine. Ces mêmes analogues de la somatostatine peuvent également être radiomarqués avec des radionucléides thérapeutiques (yttrium-90, lutétium-177) pour la radiothérapie vectorisée. Dans cet article de revue, nous faisons le point sur les principaux autres peptides radiomarqués au gallium-68, développés pour l'imagerie TEP ou la radiothérapie interne. Ces peptides peuvent être soit spécifiques de cancers surexprimant certains récepteurs, notamment des récepteurs couplés à la protéine $G$ soit être plus « généralistes » en s'adressant à l'hypoxie ou à l'angiogénèse tumorales.

\section{Analogues de la mélanocortine radiomarqués au gallium-68 : ciblage du mélanome malin}

Les mélanocortines (melanocyte-stimulating hormone [MSH]) sont des hormones synthétisées dans l'hypothalamus sous la dépendance de la leptine. Elles stimulent la synthèse de mélanine dans les cellules de la peau, les cheveux et les yeux. Ces hormones sont également impliquées dans de nombreux autres processus (synthèse des hormones stéroïdes par la corticosurrénale, obésité, inflammation, régulation cardiovasculaire et sexuelle). Les récepteurs des mélanocortines sont dénommés MC1R à MC5R. Ils sont tous couplés principalement à la protéine $\mathrm{G}$ stimulant les adénylatecyclases (synthèse d'AMP cyclique). MC1R est impliqué dans la stimulation des mélanocytes, alors que MC4R est bien connu pour être impliqué dans l'obésité. Les mélanomes (mélaniques ou non) murins [1] et humains [2] surexpriment des récepteurs pour les $\alpha$-MSH (récepteurs à mélanocortine de type 1 ou MC1R) (Fig. 1).

Les récepteurs $\mathrm{MC1R}$ sont des cibles tumorales pour l'imagerie des mélanomes et de leurs métastases. Les pionniers de ce modèle ont été Bagutti et al. [3], Bard et al. [4] et Wraight et al. [5], qui ont testé au début des années 1990 différents analogues de l' $\alpha$-MSH conjugués avec l'acide diéthylène triamine pentacétique (DTPA) et radiomarqués avec l'indium111. Les études ont été conduites chez la souris ou chez l'homme, avec des résultats variables en termes de spécificité de ciblage, mais souvent avec une forte activité rénale, comme il est souvent observé avec les petites molécules comme les peptides. Pour des applications thérapeutiques, cette forte élimination rénale laissait craindre une néphrotoxicité, si bien que de nouveaux analogues conjugués au DOTA en différentes positions ont été proposés par la suite, parmi lesquels le $\left[\mathrm{Nle}^{4}\right.$, $\left.\mathrm{ASP}^{5}, \quad \mathrm{D}-\mathrm{Phe}^{7}\right]-\alpha-\mathrm{MSH}_{4-11}$ (NAPamide), radiomarqué à l'indium-111 [6] ou au cuivre-64 [7].

Avec le gallium-68, les seules études disponibles in vivo ciblant le récepteur MC1R sont des études précliniques sur modèle murin. Cependant, la densité des récepteurs MC1R dans les mélanomes humains est beaucoup plus faible que dans le modèle de tumeur murine et les études ultérieures sur l'homme restent à faire pour déterminer la valeur diagnostique de ces traceurs. Le NAPamide-DOTA- ${ }^{68} \mathrm{Ga}$ a été développé par Froidevaux et al. [6,8], et a permis d'obtenir des images TEP de contraste élevé sur un modèle de mélanome murin B16/F1. L'autre groupe d'analogues est celui des analogues cyclisés de l' $\alpha$-MSH (CCMSH). Les études préliminaires faites, avec des analogues cyclisés de l' $\alpha$-MSH, radiomarqués avec le technétium-99m ou le rhénium-188 [9], ont montré qu'ils étaient rapidement internalisés, avec comme conséquence une rétention plus longue dans les mélanocytes. Ces analogues, stabilisés par un métal non radioactif (rhénium) et modifiés en position 11 (arginine à la place de la lysine pour limiter la rétention rénale) ont été également utilisés avec d'autres radioisotopes.

Avec le lutétium-177 et le plomb-212, dans le modèle murin $\mathrm{B} 16 / \mathrm{F} 1$, ces conjugués ont montré une excellente captation dans la tumeur et une faible captation urinaire [10,11]. Le ${ }^{68} \mathrm{Ga}$-DOTA-Re( $\left(\mathrm{Arg}^{11}\right) \mathrm{CCMSH}$ a été proposé par la même équipe [12], mais les résultats initiaux ont été décevants, notamment en raison d'une faible activité tumorale. Récemment, une méthode de préparation d'un conjugué à haute activité spécifique a permis d'obtenir, sur le même modèle murin $\mathrm{B} 16 / \mathrm{F} 1$, une très forte captation tumorale du ${ }^{68} \mathrm{Ga}$ DOTA-Re(Arg $\left.{ }^{11}\right) \mathrm{CCMSH}$ avec une excellente clairance rénale [13]. Enfin, signalons un nouveau développement par la même équipe de ces analogues cyclisés, avec l'utilisation du chélate bi-fonctionnel le CHX-A" et radiomarqués avec différents isotopes pour former le ${ }^{68} \mathrm{Ga}-\mathrm{CHX}-\mathrm{A}$ "' $\operatorname{Re}\left(\operatorname{Arg}\left({ }^{11}\right)\right) C C M S H, 1{ }^{111}$ In-CHX-A"'-Re(Arg $\left.\left({ }^{11}\right)\right) C C M S H$, ou 1 ${ }^{, 86}$ Y-CHX-A"'-Re(Arg $\left.\left({ }^{11}\right)\right) \mathrm{CCMSH}$ [14]. L'intérêt principal de ces analogues semble être la facilité de radiomarquage avec l'absence de purification, qui permet d'envisager une préparation plus aisée pour les études cliniques.

\section{Analogues bombésine-like radiomarqués au gallium- 68 et ciblage des récepteurs couplés à la protéine $G$}

L'hypothèse autocrine du développement des cancers suppose que la cellule tumorale secrète à la fois un facteur 


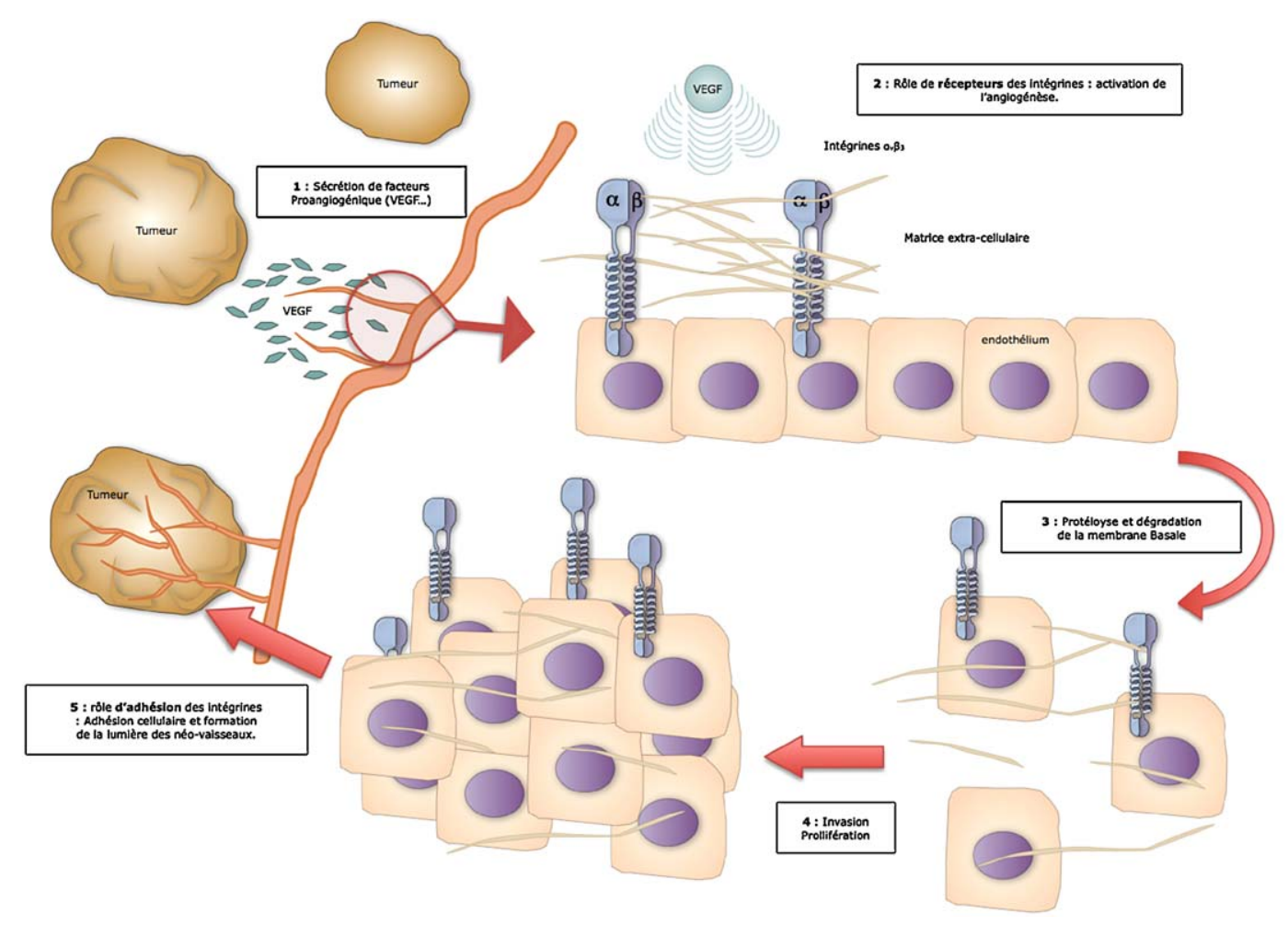

Fig. 1. Régulation de la pigmentation de la peau par $\alpha-M S H / M C 1 R$. Le message est transporté à travers la membrane grâce à l'interaction de trois protéines membranaires : les récepteurs, les protéines G et l'adenylate cyclase (CA) qui hydrolyse l'ATP en AMPc. L'AMPc se lie à une sous-unité régulatrice inhibitrice de la protéine kinase A, provoquant la dissociation de cette sous-unité catalytique et la rendant disponible. Cette sous-unité peut hydrolyser le cAMP-responsive-element (binding protein) $(\mathrm{CRE}[\mathrm{B}])$ qui va interagir avec le microphtalmia-associated transcription factor (MITF) et conduire à la régulation de l'expression des gènes de la pigmentation tyrosine-related protein I (TRP 1) et dopachrome tautomerase (TRP2).

Regulation of skin pigmentation by $\alpha-M S H / M C 1 R$.

de croissance et son récepteur apparenté, et que leur interaction provoque la prolifération tumorale. Le modèle autocrine de croissance tumorale de référence est celui du peptide libérant de la gastrine (gastrin-releasing peptide [GRP]) et de son récepteur dans les cancers pulmonaires à petites cellules [15].

La bombésine est un peptide isolé chez les amphibiens dont l'homologue chez les mammifères est le GRP. Un autre peptide bombésine-like a été identifié par la suite dans la moelle épinière chez le porc et a été appelé la neuromédine $B(\mathrm{NMB})$. Ces trois peptides de la famille du GRP exercent leurs effets en se liant à des récepteurs spécifiques présents sur des cellules du tractus digestif (notamment colon et estomac) et du système nerveux central. Il existe plusieurs sous-types de récepteurs reconnaissant les peptides de la famille GRP, parmi lesquels : NMBR pour la neuromédine $\mathrm{B}$ (neuromedin $B$ receptor ou $\mathrm{BB}_{1}$ dans la nouvelle classification), GRPR pour le GRP receptor ou $\mathrm{BB}_{2}$ et $\mathrm{BRS}$ pour les peptides bombésine-like (bombesin receptor subtype 3 ou $\mathrm{BB}_{3}$ ). Ces récepteurs sont tous des récepteurs couplés à la protéine $\mathrm{G}$ (guanine nucleotide binding protein), ayant sept domaines transmembranaires et activant différents médiateurs intracellulaires (Fig. 2). Chez l'homme, les GRPR $\left(\mathrm{BB}_{2}\right)$ sont majoritairement exprimés dans le cancer de la prostate, les gastrinomes et le cancer du sein, alors qu'une expression concomitante de GRPR $\left(\mathrm{BB}_{2}\right)$ et de $\mathrm{BRS}\left(\mathrm{BB}_{3}\right)$ a été principalement retrouvée dans le cancer du poumon à petites cellules [16].
De façon générale, l'expression de GRPR $\left(\mathrm{BB}_{2}\right)$ et/ou de BRS $\left(\mathrm{BB}_{3}\right)$ s'observe dans de nombreuses lignées cellulaires tumorales, à savoir le cancer pulmonaire à petites cellules, du pancréas, de l'estomac, de la prostate, du colon, du sein, les gliomes et les sarcomes d'Ewing.

Dans les tumeurs, les peptides GRP-like sont connus pour leurs effets mitogéniques. Ils seraient par conséquent des facteurs de croissance autocrine, secrétés par la tumeur ellemême, et impliqués dans la prolifération, la régulation de la migration des cellules tumorales et dans leur capacité d'invasion du tissu environnant. Cependant, le récepteur peut être activé par le GRP provenant de la tumeur elle-même ou d'une autre source.

La possibilité de ciblage de ces récepteurs par des analogues peptidiques GRP-like radiomarqués au gallium-68 a fait l'objet de peu de publications. L'analogue qui a été utilisé est un analogue pan-bombésine appelé $\mathrm{BZH}_{3}$, c'est-à-dire un peptide reconnaissant les trois sous-types $\mathrm{BB}_{1}, \mathrm{BB}_{2}$ et $\mathrm{BB}_{3}$. $\mathrm{Le} \mathrm{BZH}_{3}$ est un peptide DOTA-PEG 2 -[D-Tyr $\left.{ }^{6}, \mathrm{~B}-\mathrm{Ala}^{11}, \mathrm{Thi}^{13}, \mathrm{Nle}^{14}\right] \mathrm{BN}(6-14)$ amide, qui est un développement des analogues pan-bombésine $\mathrm{BZH}_{1}$ et $\mathrm{BZH}_{2}$, radiomarqués initialement au lutétium-177, à

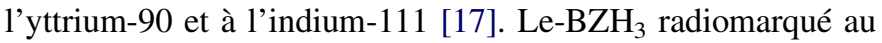
gallium-68 a été utilisé chez la souris nude avec un modèle humain de cancer du pancréas (AR4-2J). Des images de bon contraste ont été obtenues en microTEP avec un bon ratio de fixation tumeur/bruit de fond en relation avec la haute affinité du 


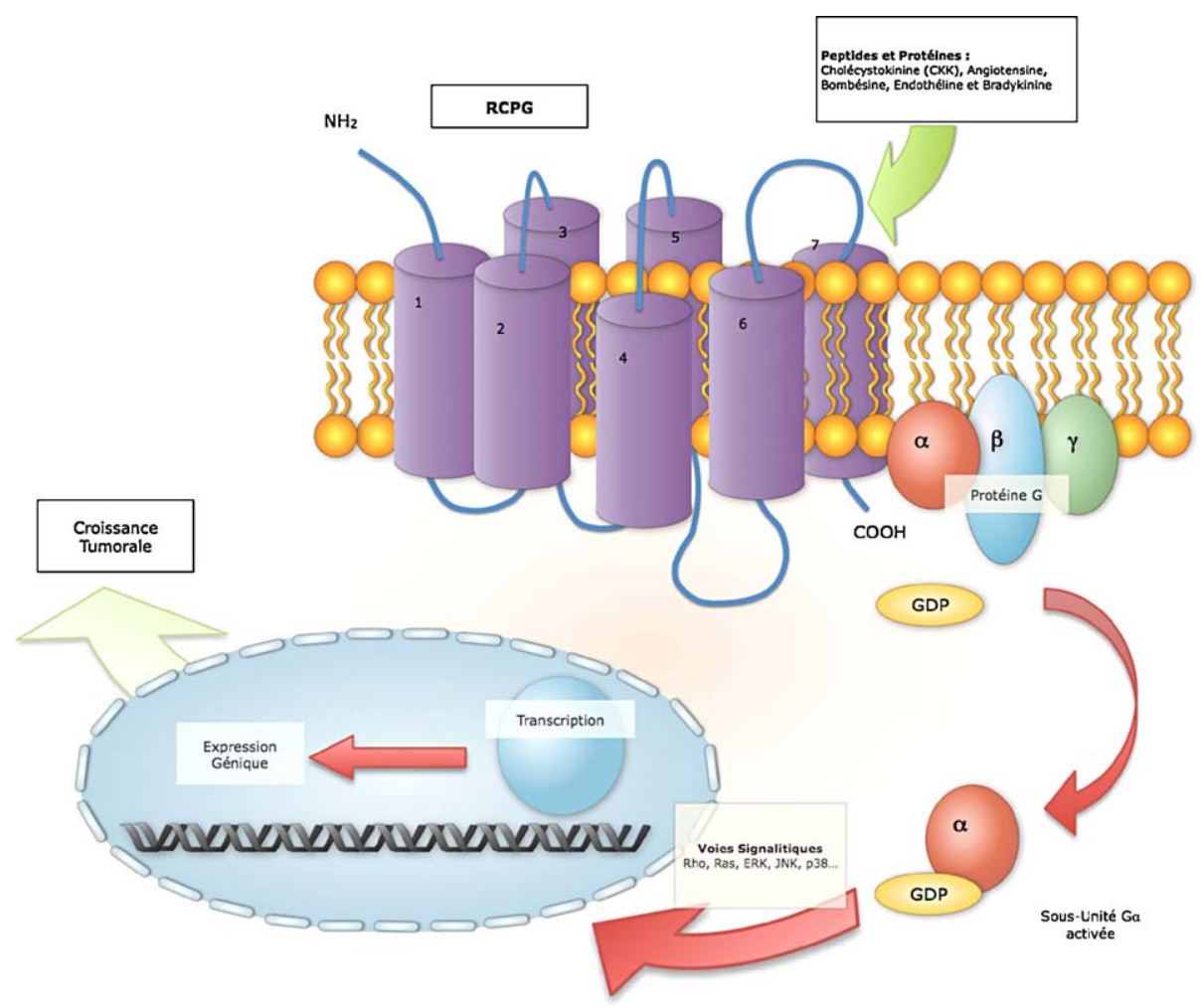

Fig. 2. Récepteur couplé à la protéine $\mathrm{G}(\mathrm{RCPG})$ et croissance tumorale. Les neuropeptides tels que le gastric-releasing peptide (GRP) et la neuromédine $\mathrm{B}$ (NMB ; bombésine-like peptide), l'endothéline, la bradykinine, la cholécystokinine (CCK) et l'angiotensine II peuvent se lier à leur récepteur couplé à la protéine G (RCPG) qui leur est apparenté, conduisant à la stimulation de la prolifération cellulaire de nombreux cancers agressifs de l'homme (notamment le cancer pulmonaire à petites cellules, le cancer du pancréas et le cancer de la prostate).

Guanine nucleotide binding protein receptor and tumour proliferation.

${ }^{68} \mathrm{Ga}^{-\mathrm{BZH}_{3}}$ pour les RGRP déterminée in vitro [18]. Plus récemment, ce même radiopharmaceutique a été utilisé en clinique chez 17 patients [19], tous porteurs de tumeurs stromales gastro-intestinales (GIST), et les résultats obtenus avec le ${ }^{68} \mathrm{Ga}$ $\mathrm{BZH}_{3}$-TEP ont été comparés à ceux obtenus avec le ${ }^{18} \mathrm{~F}$-FDGTEP. Les résultats restent peu convaincants, car sans réelle surprise dans les GIST, le FDG s'étant montré très supérieur en identifiant correctement 25/30 lésions, alors que le ${ }^{68} \mathrm{Ga}^{-\mathrm{BZH}_{3}-}$ TEP n'a pu identifier que $8 / 30$ lésions.

Parallèlement, une étude préclinique chez la souris nude avec le modèle PC-3 de cancer prostatique humain surexprimant des récepteurs bombésine-like, a été conduite en utilisant un analogue DOTA-PEG 4 (appelé DOTA-PESIN) radiomarqué soit au gallium-68, soit au lutétium-177 [20]. Le ${ }^{68} \mathrm{Ga} /{ }^{177} \mathrm{Lu}-$ DOTA-PESIN a montré in vitro une grande affinité avec les récepteurs $\mathrm{BB}_{1}$ et $\mathrm{BB}_{2}$, mais pas avec les récepteurs $\mathrm{BRS}\left(\mathrm{BB}_{3}\right)$ et le ${ }^{68} \mathrm{Ga}$-DOTA-PESIN-TEP a montré des fortes fixations tumorales une heure après l'injection.

Des études précliniques prometteuses ont été conduites avec des analogues-DOTA ciblant d'autres récepteurs couplés à la protéine $\mathrm{G}$, (autres que les récepteurs à la somatostatine qui ne sont pas envisagés dans cet article de revue), tels que la substance $\mathrm{P}$, la neurotensine et la cholécystokinine (CCK), mais radiomarquées à l'indium-111. Ces études pourraient servir de modèle pour des essais utilisant le gallium-68. À ce jour, la seule étude disponible concerne le ciblage par l'exendine-3 du récepteur du Glucagon-like peptide l(GLP-1R), qui est un récepteur couplé à la protéine $G$ surexprimé dans les insulinomes. La biodistribution de $\left[\mathrm{Lys}^{40}\left({ }^{68} \mathrm{Ga}\right.\right.$-DOTA)]Exendin-3 a été comparée à celle du même conjugué radiomarqué à l'indium-111, chez la souris nude porteuse d'un insulinome sous-cutané (INS-1). Une fixation tumorale spécifique (insulinome) mais également une fixation non spécifique pancréatique et rénale ont été observées avec les deux radioconjugués, avec cependant une fixation tumorale plus faible avec le radioconjugué marqué au gallium-68 [21].

\section{4. ${ }^{68}$ Ga-EC-métronidazole et hypoxie tumorale}

Le rôle de l'oxygénation tumorale dans la réponse à la radiothérapie est connu depuis fort longtemps. Il a été établi que l'hypoxie avait un effet protecteur des cellules tumorales vis-àvis de l'irradiation et de certaines chimiothérapies, comme par exemple la mitomycine $\mathrm{C}$ [22] et la porfiromycine [23]. L'hypoxie entraîne la transcription du facteur $1 \alpha$, conduisant à l'expression de gènes qui participent à la réponse adaptative de la cellule à l'hypoxie. Une de ces réponses est l'augmentation de la vascularisation tumorale via le facteur de croissance vasculaire endothéliale (VEGF) [24]. On peut également observer une sélection de cellules mutantes pour le gène $p 53$, conduisant au blocage des mécanismes d'apoptose et à une instabilité génétique qui peut conduire à l'amplification de gènes impliqués dans la résistance à certaines chimiothérapies $[25,26]$. Ces différents mécanismes accroissent les capacités de 
survie et de prolifération des cellules tumorales. Ainsi, l'hypoxie pourrait être un facteur important de résistance thérapeutique, en particulier à la radiothérapie de nombreux types de tumeurs solides. Plusieurs études ont montré une corrélation entre la présence d'une fraction de cellules hypoxiques au sein des tumeurs et un moins bon contrôle local par la radiothérapie de gliomes [27], de tumeurs du col utérin [28], de tumeurs de la tête et du cou [29,30], et de sarcomes des tissus mous [31]. Des cellules hypoxiques existeraient dans à peu prés toutes les tumeurs solides, mais en proportion variable dans le temps et en fonction du type de tumeur et avec une distribution intratumorale hétérogène même pour des tumeurs de nature identique ou de même présentation clinique et radiologique.

Chapman J.D. et al. ont été les premiers à avoir proposé l'usage in vivo des nitroimidazoles comme marqueurs bioréductibles de l'hypoxie tissulaire et comme sensibilisant à la radiothérapie des tumeurs hypoxiques [32]. En conditions hypoxiques, les nitroimidazoles sont réduits en métabolites réactifs intermédiaires par des 1-électron réductases intracellulaires, avec un taux de production d'autant plus important que l'hypoxie est profonde. Cette production peut atteindre des ratios 30 à 40 fois plus élevés que ceux observés dans les cellules aérobies, ce qui crée un gradient très favorable à la détection spécifique des cellules hypoxiques. Ces métabolites intermédiaires forment des liaisons covalentes avec des groupements thiols des protéines intracellulaires ; ils sont alors retenus et s'accumulent dans les cellules hypoxiques viables. Les complexes métabolites réduits-protéines intracellulaires peuvent être détectés par de nombreuses techniques dont l'imagerie scintigraphique.

Le chef de file pour l'imagerie scintigraphique de l'hypoxie est le 2-nitroimidazole. Il a été radiomarqué avec le fluor-18 ( $\left.{ }^{18} \mathrm{~F}-\mathrm{FMISO}\right)[33,34]$, l'iode-123 et -131 (iodinated azomycine arabinoside [IAZA]) [35-37], et le technétium 99m. Plus récemment, le métronidazole a été radiomarqué avec succès avec du gallium-68 en utilisant l'éthylène-dicystéine comme chélateur [38]. La captation du traceur en TEP a été corrélée à la mesure de la pression partielle en $\mathrm{O}_{2}$ intratumorale chez le rat porteur d'une tumeur mammaire. Le ${ }^{68} \mathrm{Ga}-\mathrm{EC}-\mathrm{MN}$ a permis de visualiser clairement les tumeurs hypoxiques, mais aucune autre étude n'a été publiée depuis chez l'animal ou chez l'homme avec ce radioconjugué.

\section{5. ${ }^{68}$ Gallium-DOTA-RGD peptides et angiogenèse}

L'angiogenèse, processus de développement de nouveaux vaisseaux sanguins, est une condition préalable au développement de tumeurs, et par conséquent, constitue un point important dans le contrôle de la progression du cancer. L'angiogenèse tumorale comporte plusieurs étapes dont :

- une réponse chimiotactique et mitogénique des cellules endothéliales aux facteurs de croissance angiogéniques, notamment par le vascular endothélial growth factor (VEGF) mais aussi par de nombreux autres facteurs ;
- une dégradation protéolytique de la matrice extracellulaire (ensemble de macromolécules extracellulaires formant le tissu de soutien des cellules et de l'endothélium) ;

- une modulation des interactions entre les cellules endothéliales avec la matrice extracellulaire médiée par les récepteurs des intégrines.

Les intégrines sont des protéines transmembranaires, constituées d'une sous-unité alpha et d'une sous-unité bêta. Elles sont impliquées dans les phénomènes d'adhésion cellulaire en se liant aux molécules de la matrice extracellulaire. Une de ces intégrines, le $\alpha_{\mathrm{v}} \beta_{3}$, a récemment été ciblée pour de nouvelles thérapies antitumorales, car c'est une protéine transmembranaire fortement surexprimée dans les cellules endothéliales angiogéniques. Son rôle dans la croissance tumorale, l'invasion et le développement de métastase a été largement démontré dans de nombreuses tumeurs (Fig. 3).

La cible que représente l'intégrine $\alpha_{v} \beta_{3}$ a été utilisée en TEP pour évaluer l'activité de néovascularisation associée à la croissance tumorale. Plusieurs travaux ont permis d'améliorer la stabilité et l'efficacité du ciblage de radiomarqueurs de l'intégrine $\left\langle_{\mathrm{v}} \mathbb{R}_{3}\right.$ pour la TEP. À ce jour, le plus prometteur de ces marqueurs des intégrines reste le ${ }^{18} \mathrm{~F}$-galacto-RGD. Les premiers essais cliniques chez des volontaires sains et un nombre limité de patients atteints de cancer ont révélé que ce traceur peut être administré sans danger aux patients, et ont montré la possibilité d'une évaluation de l'activité angiogénique in vivo avec une bonne corrélation de l'expression de $\alpha_{v} \beta_{3}$ et de l'imagerie TEP au ${ }^{18}$ F-galacto-RGD.

Il n'y a pas à ce jour d'essais cliniques publiés avec un peptide RGD marqué au gallium-68. Les publications in vitro et in vivo précliniques sont d'ailleurs très récentes et peu nombreuses. Un article relate la première expérience avec le ${ }^{68}$ Ga-DOTA-RGD et compare sa biodistribution chez la souris nude porteuse de mélanome M21 ( $\alpha_{\mathrm{v}} \beta_{3}$ positive $)$ vs des animaux porteurs d'un mélanome $\alpha_{v} \beta_{3}$ négative [39]. Le ${ }^{68} \mathrm{Ga}-$ DOTA-RGD a permis de visualiser spécifiquement les tumeurs $\left\langle_{\mathrm{v}} \mathbb{R}_{3}\right.$ positives, mais les rapports tumeur/activité sanguine étaient plus faibles avec le ${ }^{68} \mathrm{Ga}$-DOTA-RGD vs ${ }^{111}$ In-DOTARGD et ${ }^{18}$ F-Galacto-RGD. D'autres peptides RGD cycliques (RGDyK) ont été chélatés avec l'acide 1,4,7-triazacyclononane-1,4,7-triacetique (NOTA), avec la volonté d'identifier un conjugué avec une forte captation tumorale et une extraction urinaire rapide, pour faire baisser rapidement l'activité sanguine. Les dimères RGD tels que le ${ }^{68}$ Ga-NOTA-RGD2 [40], le ${ }^{68}$ Ga-NOTA-P 4 -RGD2 et le ${ }^{68}$ Ga-NOTA-Gly3-RGD2 [41] ont montré qu'ils avaient la plus grande spécificité et affinité pour les intégrines, et en conséquence les plus fortes intensités de fixation tumorale, tout en ayant une élimination urinaire rapide. Une autre équipe a développé une autre approche dans laquelle le RGDyK a été conjugué avec le 2-p-isothiocyanatobenzyl-NOTA (SCN-Bz-NOTA) et radiomarqué au gallium-68 [42]. Un ${ }^{68} \mathrm{Ga}-\mathrm{SCN}-\mathrm{Bz}-\mathrm{NOTA-RGD} \mathrm{a}$ pu être radiosynthétisé avec un haut rendement de marquage (89\%), et a permis d'imager spécifiquement en microTEP les tissus $\alpha_{v} \beta_{3}$ positifs (muscles et tumeurs) tout en montrant une 


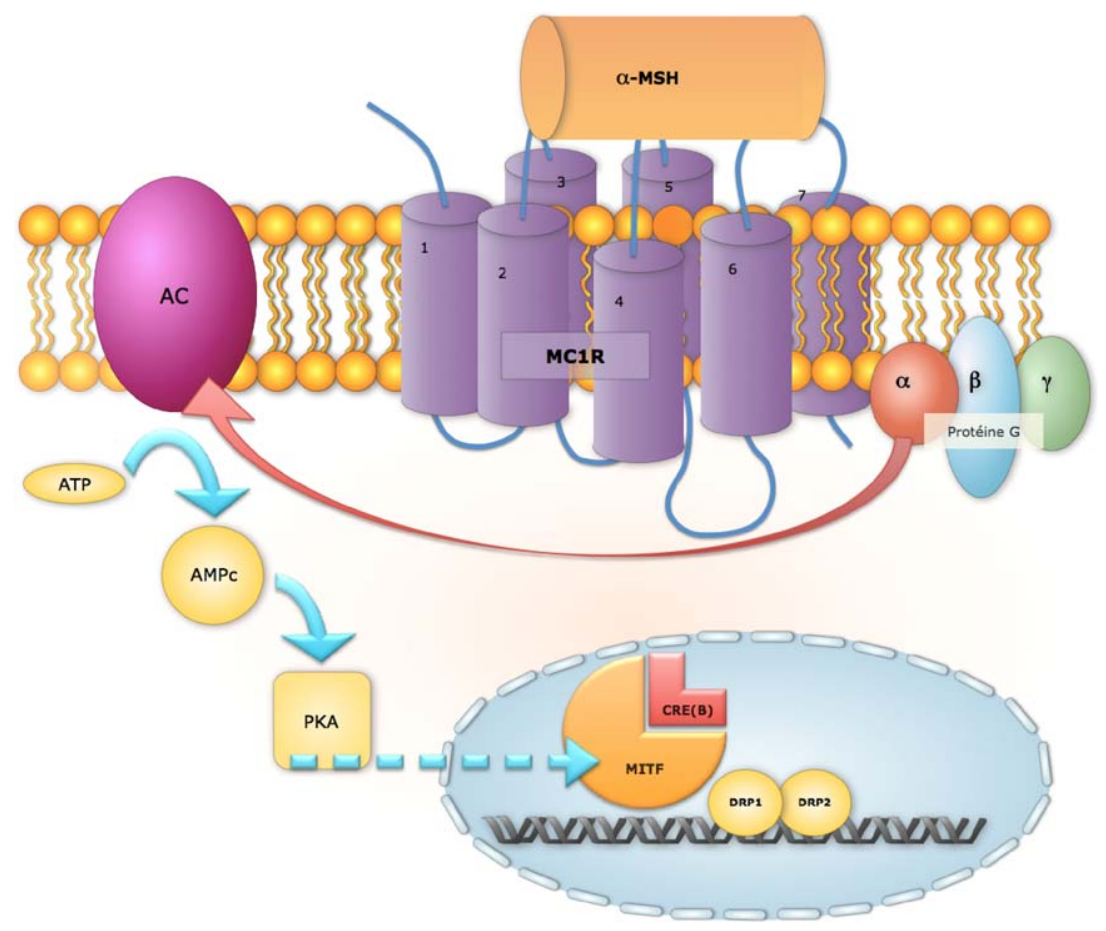

Fig. 3. Rôles des intégrines dans l'angiogénèse. Les cellules endothéliales expriment différentes familles d'intégrines (chaque famille étant spécifique de molécules précises) qui permettent leur attachement aux protéines de la matrice extracellulaire. Ces intégrines peuvent détecter les modifications du microenvironnement, et l'interpréter comme un signal à l'origine de processus cellulaire tel que l'angiogénèse. Ainsi, la présence de VEGF dans la matrice extracellulaire peut initier l'angiogénèse. La matrice extracellulaire est alors dégradée par des protéinases, pour permettre l'invasion et la prolifération des cellules endothéliales. Par la suite, les interactions entre intégrines et constituants de la matrice extracellulaire vont conduire au réarrangement du cytosquelette des cellules endothéliales et à la formation de néovaisseaux.

Roles of integrins in angiogenesis process.

élimination urinaire rapide. Signalons enfin le développement très prometteur d'un hétérodimère reconnaissant à la fois les intégrines $\alpha_{v} \beta_{3}$ et les récepteurs du GRP, chélaté avec le NOTA et radiomarqué au gallim-68 [43]. Le rationnel supportant le développement d'un tel hétérodimère ${ }^{68} \mathrm{Ga}$-NOTA-RGD-BBN est la variabilité de l'expression des cibles tumorales (GRPR et intégrines $\alpha_{v} \beta_{3}$ ) tout au long du processus de croissance et d'invasion tumorales et de développement des métastases. Ce conjugué a été testé avec succès chez la souris porteuse de tumeur prostatique humaine $\mathrm{PC}-3$ et de mélanome humain MDA-MB-435. En effet, la fixation tumorale du ${ }^{68}$ Ga-NOTA-RGD-BBN était significativement plus importante que celle du ${ }^{68} \mathrm{Ga}$-NOTA-RGD ou celle du ${ }^{68} \mathrm{Ga}$-NOTA-BBN, avec une clairance rapide des trois traceurs.

\section{Autres modèles}

Sans être exhaustif, nous signalons encore la possibilité d'imager avec le gallium-68:

- la P-glycoprotéine (Pgp), produit de la surexpression du gène de multirésistance (MDR1) [44] ;

- le transport intra-tumoral d'acides aminés avec une ${ }^{68} \mathrm{Ga}-\mathrm{L}-$ Tyrosine $\left({ }^{68} \mathrm{Ga}-\mathrm{DO}_{2} \mathrm{~A}-(\mathrm{OBu}-\mathrm{L}-\mathrm{tyr})_{2}\right)$ pour l'imagerie TEP des tumeurs cérébrales en particulier [45] ;

- les altérations génétiques à l'origine des cancers avec des ${ }^{68}$ Ga-DOTA-oligonucléotides [46] ;
- les récepteurs à activité tyrosine kinase tel que le récepteur à l'HER2 (EGF-R) avec des petites protéines affibody radiomarquées [47].

\section{Conclusion}

Malgré ces perspectives encourageantes pour l'utilisation de ${ }^{68} \mathrm{Ga}$-TEP, il n'y a que pas ou très peu d'études cliniques avec des peptides radiomarqués au gallium-68 en dehors des analogues de la somatostatine. Il faut de plus souligner qu'aucun industriel n'a encore obtenu une autorisation de commercialisation d'un générateur ${ }^{68} \mathrm{Ge} /{ }^{68} \mathrm{Ga}$. Cela signifie que les nombreux composés radiomarqués au ${ }^{68} \mathrm{Ga}$ envisagés dans cet article ne seront pas disponibles avant encore de nombreuses années en tant que produits radiopharmaceutiques, et commercialisés selon le modèle du technétium- $99 \mathrm{~m}$ pour une utilisation à grande échelle en clinique. Sans autorisation de mise sur le marché, les études cliniques resteront dans le domaine de la recherche, difficiles à financer et à réaliser étant donné le nombre de molécules, alors que ces essais cliniques sont absolument nécessaires pour déterminer les qualités cliniques de ces molécules.

\section{Conflit d'intérêt}

Aucun. 


\section{Références}

[1] Tatro JB, Reichlin S. Specific receptors for alpha-melanocyte-stimulating hormone are widely distributed in tissues of rodents. Endocrinology 1987;121:1900-7.

[2] Siegrist W, Solca F, Stutz S, Giuffre L, Carrel S, Girard J, et al. Characterization of receptors for alpha-melanocyte-stimulating hormone on human melanoma cells. Cancer Res 1989;49:6352-8.

[3] Bagutti C, Stolz B, Albert R, Bruns C, Pless J, Eberle AN. [111In]-DTPAlabeled analogues of alpha-melanocyte-stimulating hormone for melanoma targeting: receptor binding in vitro and in vivo. Int $\mathrm{J}$ Cancer 1994;58:749-55.

[4] Bard DR, Knight CG, Page-Thomas DP. A chelating derivative of alphamelanocyte stimulating hormone as a potential imaging agent for malignant melanoma. Br J Cancer 1990;62:919-22.

[5] Wraight EP, Bard DR, Maughan TS, Knight CG, Page-Thomas DP. The use of a chelating derivative of alpha melanocyte stimulating hormone for the clinical imaging of malignant melanoma. Br J Radiol 1992;65:112-8.

[6] Froidevaux S, Calame-Christe M, Tanner HE, Berle AN. Melanoma targeting with DOTA-alpha-melanocyte-stimulating hormone analogs: structural parameters affecting tumor uptake and kidney uptake. J Nucl Med 2005;46:887-95.

[7] Cheng Z, Xiong Z, Subbarayan M, Chen X, Gambhir SS. 64Cu-labeled alpha-melanocyte-stimulating hormone analog for microPET imaging of melanocortin 1 receptor expression. Bioconjug Chem 2007;18: 765-72.

[8] Froidevaux S, Calame-Christe M, Schuhmacher J, Tanner H, Saffrich R, Henze M, et al. A gallium-labeled DOTA-alpha-melanocyte- stimulating hormone analog for PET imaging of melanoma metastases. J Nucl Med 2004;45:116-23.

[9] Miao Y, Owen NK, Whitener D, Gallazzi F, Hoffman TJ, Quinn TP. In vivo evaluation of $188 \mathrm{Re}$-labeled alpha-melanocyte stimulating hormone peptide analogs for melanoma therapy. Int J Cancer 2002;101:480-7.

[10] Miao Y, Shelton T, Quinn TP. Therapeutic efficacy of a 177Lu-labeled DOTA conjugated alpha-melanocyte-stimulating hormone peptide in a murine melanoma-bearing mouse model. Cancer Biother Radiopharm 2007;22:333-41.

[11] Miao Y, Hylarides M, Fisher DR, Shelton T, Moore H, Wester DW, et al. Melanoma therapy via peptide-targeted \{alpha\}-radiation. Clin Cancer Res 2005;11:5616-21.

[12] Wei L, Miao Y, Gallazzi F, Quinn TP, Welch MJ, Vavere AL, et al. Gallium-68-labeled DOTA-rhenium-cyclized alpha-melanocyte-stimulating hormone analog for imaging of malignant melanoma. Nucl Med Biol 2007;34:945-53.

[13] Cantorias MV, Figueroa SD, Quinn TP, Lever JR, Hoffman TJ, Watkinson LD, et al. Development of high-specific-activity (68)Ga-labeled DOTArhenium-cyclized alpha-MSH peptide analog to target $\mathrm{MC1}$ receptors overexpressed by melanoma tumors. Nucl Med Biol 2009;36:505-13 .

[14] Wei L, Zhang X, Gallazzi F, Miao Y, Jin X, Brechbiel MW, et al. Melanoma imaging using (111)In-(86)Y- and (68)Ga-labeled CHX-A"$\operatorname{Re}(\operatorname{Arg} 11)$ CCMSH. Nucl Med Biol 2009;36:345-54.

[15] Cuttitta F, Carney DN, Mulshine J, Moody TW, Fedorko J, Fischler A, et al. Bombesin-like peptides can function as autocrine growth factors in human small-cell lung cancer. Nature 1985;316:823-6.

[16] Zhang X, Cai W, Cao F, Schreibmann E, Wu Y, Wu JC, et al. 18F-labeled bombesin analogs for targeting GRP receptor-expressing prostate cancer. J Nucl Med 2006;47:492-501.

[17] Zhang H, Chen J, Waldherr C, Hinni K, Waser B, Reubi JC, et al. Synthesis and evaluation of bombesin derivatives on the basis of pan-bombesin peptides labeled with indium-111, lutetium-177, and yttrium-90 for targeting bombesin receptor-expressing tumors. Cancer Res 2004;64:670715.

[18] Schuhmacher J, Zhang H, Doll J, Macke HR, Matys R, Hauser H, et al. GRP receptor-targeted PET of a rat pancreas carcinoma xenograft in nude mice with a 68Ga-labeled bombesin(6-14) analog. J Nucl Med 2005; 46:691-9.

[19] Dimitrakopoulou-Strauss A, Hohenberger P, Haberkorn U, Macke HR, Eisenhut M, Strauss LG. 68Ga-labeled bombesin studies in patients with gastrointestinal stromal tumors: comparison with 18F-FDG. J Nucl Med 2007;48:1245-50.

[20] Zhang H, Schuhmacher J, Waser B, Wild D, Eisenhut M, Reubi JC, et al. DOTA-PESIN, a DOTA-conjugated bombesin derivative designed for the imaging and targeted radionuclide treatment of bombesin receptor-positive tumours. Eur J Nucl Med Mol Imaging 2007;34:1198-208.

[21] Brom M, Oyen WJ, Joosten L, Gotthardt M, Boerman OC. (68)Galabelled exendin-3, a new agent for the detection of insulinomas with PET. Eur J Nucl Med Mol Imaging 2010;37:46-8. doi:10.1007/s00259009-1363-y.

[22] Rockwell S. Effect of some proliferative and environmental factors on the toxicity of mitomycin C. Int J Cancer 1986;38:229-35.

[23] Rockwell S, Keyes SR, Sartorelli AC. Preclinical studies of porfiromycine as an adjunct to radiotherapy. Radiat Res 1988;116:100-13.

[24] Hlatky L, Tsionou C, Hanhfeldt P, Coleman CN. Mammary fibroblasts may influence breast tumor angiogenesis, via hypoxia-induced vascular endothelial growth factor upregulation and protein expression. Cancer Res 1994;54:6083-6.

[25] Rice GC, Hoy C, Schimke RT. Transient hypoxia enhances the frequency of dihydrofolate reductase gene amplification in Chinese hamster ovary cells. Proc Natl Acad Sci USA 1986;83:5978-82.

[26] Rice GC, Ling V, Schimke RT. Frequencies of independent and simultaneous selection of Chinese hamster cells for methotrexate and doxorubicin (adriamycin) resistance. Proc Natl Acad Sci USA 1987;84:9261-4.

[27] Teicher BA. Physiologic mechanisms of therapeutic resistance. Hematol Oncol Clin North Am 1995;9:475-506.

[28] Hockel M, Schlenger K, Aral B, Mitze M, Schaffer U, Vaupel P. Association between tumor hypoxia and malignant progression in advanced cancer of the uterine cervix. Cancer Res 1996;56:4509-15.

[29] Brizel DM, Sibley GS, Prosnitz LR, Scher RL, Dewhirst MW. Tumor hypoxia adversely affects the prognosis of carcinoma of the head and neck. Int J Radiat Oncol Biol Phys 1997;38:285-9.

[30] Gatenby RA, Kessler HB, Rosenblum JS, Coia LR, Moldofsky PJ, Hartz $\mathrm{WH}$, et al. Oxygen distribution in squamous cell carcinoma metastases and its relationship to outcome of radiation therapy. Int J Radiat Oncol Biol Phys 1988;14(5):831-8.

[31] Brizel DM, Scully SP, Harrelson JM, Layfield LJ, Bean JM, Prosnitz LR, et al. Tumor oxygenation predicts for the likelihood of distant metastases in human soft tissue sarcoma. Cancer Res 1996;56:941-3.

[32] Chapman JD, Franko AJ, Sharplin J. A marker for hypoxic cells in tumours with potential clinical applicability. Br J Cancer 1981;43:546-50.

[33] Grierson JR, Link JM, Mathis CA, Rasey JS, Krohn KA. A radiosynthesis of fluorine-18 fluoromisonidazole. J Nucl Med 1989;30:343-50.

[34] Groshar D, McEwan AJ. Parliament, MBal., e. Imaging tumor hypoxia and tumor perfusion. J Nucl Med 1993;34:885-8.

[35] Cherif A, Wallace S, Yang DJ, Newman RA, Harrod VL, Nornoo A, et al. Development of new markers for hypoxic cells: [131I]Iodomisonidazole and [131I]Iodoerythronitroimidazole. J Drug Target 1996;4:31-9.

[36] Melo T, Duncan J, Ballinger JR, Rauth AM. BRU59-21, a secondgeneration Tc-99m-labeled 2-nitroimidazole for imaging hypoxia in tumors. J Nucl Med 2000;41:169-76.

[37] Parliament MB, Chapman JD, Urtasun. RCal., e. Non-invasive assessment of human tumour hypoxia with 123I-iodoazomycin arabinoside: preliminary report of a clinical study. Br J Cancer 1992;65:90-5.

[38] Ito M, Yang DJ, Mawlawi O, Mendez R, Oh CS, Azhdarinia A, et al. PET and planar imaging of tumor hypoxia with labeled metronidazole. Acad Radiol 2006;13:598-609.

[39] Decristoforo C, Hernandez Gonzalez I, Carlsen J, Rupprich M, Huisman M, Virgolini I, et al. 68Ga- and 111In-labelled DOTA-RGD peptides for imaging of alphavbeta3 integrin expression. Eur J Nucl Med Mol Imaging 2008;35:1507-15.

[40] Li ZB, Chen K, Chen X. (68)Ga-labeled multimeric RGD peptides for microPET imaging of integrin alphavbeta3 expression. Eur J Nucl Med Mol Imaging 2008;35:1100-8.

[41] Liu Z, Niu G, Shi J, Liu S, Wang F, Liu S, et al. (68)Ga-labeled cyclic RGD dimers with Gly3 and PEG4 linkers: promising agents for tumor integrin alphavbeta3 PET imaging. Eur J Nucl Med Mol Imaging 2009;36:947-57. 
[42] Jeong JM, Hong MK, Chang YS, Lee YS, Kim YJ, Cheon GJ, et al. Preparation of a promising angiogenesis PET imaging agent: 68Galabeled c(RGDyK)-isothiocyanatobenzyl-1,4,7-triazacyclononane-1,4,7triacetic acid and feasibility studies in mice. J Nucl Med 2008;49:830-6.

[43] Liu Z, Niu G, Wang F, Chen X. 68Ga-labeled NOTA-RGD-BBN peptide for dual integrin and GRPR-targeted tumor imaging. Eur J Nucl Med Mol Imaging 2009;36:1483-94.

[44] Sharma V, Prior JL, Belinsky MG, Kruh GD, Piwnica-Worms D. Characterization of a $67 \mathrm{Ga} / 68 \mathrm{Ga}$ radiopharmaceutical for SPECT and PET of MDR1 P-glycoprotein transport activity in vivo: validation in multidrugresistant tumors and at the blood-brain barrier. J Nucl Med 2005;46:354-64.
[45] Burchardt C, Riss PJ, Zoller F, Maschauer S, Prante O, Kuwert T, et al. [(68)Ga]Ga-DO(2)A-(OBu-1-tyr)(2): synthesis, (68)Ga-radiolabeling and in vitro studies of a novel (68)Ga-DO(2)A-tyrosine conjugate as potential tumor tracer for PET. Bioorg Med Chem Lett 2009;19:3498501.

[46] Roivainen A, Tolvanen T, Salomaki S, Lendvai G, Velikyan I, Numminen $\mathrm{P}$, et al. 68Ga-labeled oligonucleotides for in vivo imaging with PET. J Nucl Med 2004;45:347-55.

[47] Ren G, Zhang R, Liu Z, Webster JM, Miao Z, Gambhir SS, et al. A 2-helix small protein labeled with $68 \mathrm{Ga}$ for PET imaging of HER2 expression. J Nucl Med 2009;50:1492-9. 